\title{
HUREWICZ ISOMORPHISM THEOREM FOR STEENROD HOMOLOGY
}

\author{
Y. KODAMA AND A. KOYAMA
}

\begin{abstract}
For a pointed compactum $(X, x)$, a natural homomorphism $\xi_{n}$ from the Quigley's approaching group $\underline{\underline{\pi}}_{n}(X, x)$ to the Steenrod homology group ${ }^{3} H_{n+1}(X)$ is defined. A shape theoretical condition under which $\xi_{n}$ is an isomorphism is obtained. For every pointed $S^{n}$-like continuum $(X, x), \xi_{n}$ is an isomorphism for $n \neq 2$ and $\xi_{2}$ is an isomorphism if and only if $X$ is movable.
\end{abstract}

1. Introduction. Let $X$ be a compact metric space and let $x \in X$. Denote by ${ }^{s} H_{n}(X)$ the $n$-dimensional Steenrod homology group of $X$ defined by Steenrod [14] and by $\underline{\underline{\pi}}_{n}(X, x)$ the $n$-dimensional approaching group of $(X, x)$ defined by Quigley [13]. For each $n$, there exists a natural homomorphism $\xi_{n}$ : $\underline{\pi}_{n}(X, x) \rightarrow{ }^{s} H_{n+1}(X)$. The purpose of this note is to establish a shape theoretical condition for $X$ under which $\xi_{n}$ is an isomorphism.

Throughout the paper all spaces are metrizable and maps are continuous. We denote by $J$ the directed set of nonnegative integers.

2. ( $m, n)$-movability. Let $(K, v)$ be a pointed polyhedron and let $(Y, y)$ be a pointed space. For $k \in J$, a map $f:(Y, y) \rightarrow(K, v)$ is said to be $k$-deformable if there exists a map $g:(Y, y) \rightarrow(K, v)$ such that $f \simeq g$ rel $\{y\}$ and $g(Y)$ is contained in a combinatorial $k$-skeleton of a triangulation of $K$. For a pair $(n, r), n, r \in J$, a pointed compactum $(X, x)$ is said to be $(m, n)$-movable if there exists an inverse sequence $\left\{\left(X_{i}, x_{i}\right), \pi_{i j}\right\}$ consisting of pointed finite polyhedra $\left(X_{i}, x_{i}\right), i \in J$, and bonding maps $\pi_{i j}:\left(X_{j}, x_{j}\right) \rightarrow\left(X_{i}, x_{i}\right), i<j$, $i, j \in J$, satisfying the following conditions:

(2.1) $(X, x)=\lim _{\leftarrow}\left(X_{i}, x_{i}\right)$.

(2.2) For every $i \in J$, there exists $j \in J, j \geqslant i$, such that for every $k \in J$, $k \geqslant i$, and every $n$-deformable map $f:\left(S^{m}, s\right) \rightarrow\left(X_{j}, x_{j}\right)$, where $\left(S^{m}, s\right)$ is a pointed $m$-sphere, there exists an $n$-deformable map $g:\left(S^{m}, s\right) \rightarrow\left(X_{k}, x_{k}\right)$ such that $\pi_{i j} f \simeq \pi_{i k} g$ rel $s$.

For a pointed compactum $(X, x)$, denote by $\operatorname{Sh}(X, x)$ the shape of $(X, x)$ defined by Borsuk [2]. The $(m, n)$-movability of $(X, x)$ is a hereditary shape invariant in the sense of Borsuk, that is, we have

Received by the editors February 15, 1978 and, in revised form, June 13, 1978.

AMS (MOS) subject classifications (1970). Primary 54F99; Secondary 55B99, 55E99.

Key words and phrases. Shape, movability, Steenrod homology, Hurewicz isomorphism theorem. 
TheOREM 1. Let $(X, x)$ and $(Y, y)$ be pointed compacta. If $\operatorname{Sh}(X, x) \geqslant$ $\operatorname{Sh}(Y, y)$ and $(X, x)$ is $(m, n)$-movable, then $(Y, y)$ is $(m, n)$-movable.

The proof is given by a standard technique (cf. [7, Theorem 1]) and we omit it.

For $n \in J$, the pointed $n$-movability of $(X, x)$ is defined in a similar way to one given by Borsuk [1], that only the category of pointed compacta is considered. The following lemma is obvious from [7].

Lemma 1. A pointed n-movable compactum is $(m, n)$-movable for every $m \in J$.

Since the pointed movability (cf. [2, p. 166]) implies the pointed $n$-movability for every $n \in J$, we have

COROllary 1. A pointed movable compactum is $(m, n)$-movable for $m$, $n \in J$.

EXAMPLE 1. Let $n \in J, n>0$. Let $\left\{X_{i}, p_{i, i+1}\right\}$ be an inverse sequence consisting of $n$-spheres $X_{i}, i \in J$, such that each bonding map $p_{i, i+1}: X_{i+1} \rightarrow$ $X_{i}$ is of degree 2. Let $X(n)$ be the limit space of $\left\{X_{i}\right\}$ and $x \in X(n)$. Then we have

(2.3) if $n \neq 2,(X(n), x)$ is $(n+1, n)$-movable,

(2.4) $(X(2), x)$ is not $(3,2)$-movable.

To see (2.3) it is enough to note that $\pi_{2}\left(S^{1}\right)=0$ and $\pi_{n+1}\left(S^{n}\right)=Z_{2}$ for $n \geqslant 3$, where $\pi_{i}(Y)$ is the $i$-homotopy group of $Y$. (Note that $X(1)$ is $(k, 1)$-movable for each $k>1$.)

Assertion (2.4) follows from $\pi_{3}\left(S^{2}\right)=Z$ (see [6, Chapter VI, Lemma 1.2]) and the definition of the $(3,2)$-movability.

Since $(X(n), x)$ is not pointed $n$-movable, the converse assertion of Lemma 1 or Corollary 1 does not generally hold.

3. Hurewicz isomorphism theorem. For a compactum $X$, let ${ }^{s} H_{n}(X)$ be the homology group of the regular $n$-cycles of $X$ defined by N. E. Steenrod [14]. A beautiful description of ${ }^{s} H_{n}(X)$ was given by J. Milnor [11]. For a pointed compactum $(X, x)$, Quigley [13] defined the approaching group $\underline{\underline{\pi}}_{n}(X, x)$. To define a natural homomorphism $\xi_{n}: \underline{\pi}_{n}(X, x) \rightarrow^{s} H_{n+1}(X)$, consider $X$ as a subset of the Hilbert cube $Q$. For an element $\alpha \in \underline{\pi}_{n}(X, x)$, let $f: R^{+} \times$ $\left(S^{n}, s\right) \rightarrow(Q, x)$ be an approaching $n$-mapping representing in the sense of Quigley [13], where $R^{+}=\{t: 0 \leqslant t<\infty\}$. Let $D^{n+1}$ be an $(n+1)$-ball whose boundary is $S^{n}$ and put $K=\{0\} \times D^{n+1} \cup R^{+} \times S^{n}$. Let $g: K \rightarrow Q$ be an extension of $f$. Since $K$ is an infinite $(n+1)$-cycle, the triple $(K, f, K)$ is an infinite $(n+1)$-cycle regular to $X$ in the sense of Steenrod [14, p. 837]. Let $\beta$ be the element of ${ }^{s} H_{n+1}(X)$ represented by $(K, f, K)$. Obviously $\beta$ is uniquely determined by the element $\alpha$. Define $\xi_{n}: \underline{\underline{\pi}}_{n}(X, x) \rightarrow{ }^{s} H_{n+1}(X)$ by $\xi_{n}(\alpha)=\beta$. It is easy to show that $\xi_{n}$ is a natural homomorphism. 
THEOREM 2. Let $(X, x)$ be a pointed compactum and let $n \in J, n>1$. If $(X, x)$ is $(n+1, n)$-movable and approximatively $k$-connected for $k=0$, $1, \ldots, n-1[2, p .145]$, then $\xi_{n}: \underline{\underline{\pi}}_{n}(X, x) \rightarrow{ }^{s} H_{n+1}(X)$ is an isomorphism.

Proof. Let $\left\{\left(X_{i}, x_{i}\right), p_{i, i+1}\right\}$ be an inverse sequence consisting of pointed polyhedra such that $(X, x)=\lim \left\{\left(X_{i}, x_{i}\right), p_{i, i+1}\right\}$. Let $\left\{H_{k}\left(X_{i}\right)\right\}$ be the inverse sequence consisting of the $k$-homology groups. Similarly let $\left\{\pi_{k}\left(X_{i}, x_{i}\right)\right\}$ be the inverse sequence consisting of $k$-homotopy groups of $\left(X_{i}, x_{i}\right), i \in J$. From the proofs of [14, Theorem 7], [11, Theorem 4] and [15, Theorem 2] it is seen that the homomorphism $\xi_{n}$ induces homomorphisms $\mu$ and $\eta_{n}$ such that the following diagram is commutative,

$$
\begin{aligned}
& 0 \rightarrow \lim _{\leftarrow}^{(1)}\left\{\pi_{n+1}\left(X_{i}, x_{i}\right)\right\} \rightarrow \underline{\underline{\pi}}_{n}(X, x) \rightarrow \pi_{n}(X, x) \rightarrow 0 \\
& \downarrow \mu \quad \downarrow \xi_{n} \quad \downarrow \eta_{n} \\
& 0 \rightarrow \lim _{\leftarrow}^{(1)}\left\{H_{n+1}\left(X_{i}\right)\right\} \rightarrow^{s} H_{n+1}(X) \rightarrow \check{H}_{n}(X) \rightarrow 0
\end{aligned}
$$

Here $\check{H}_{n}(X)$ is the Čech $n$-homology group of $X, \underline{\pi}_{n}(X, x)$ is the $n$th fundamental group defined by Borsuk [2, Chapter XII] and $\lim ^{(1)}$ is the first derived functor of the inverse limit functor $\lim$. The homomorphism $\mu$ is induced by the Hurewicz homomorphism $\mu_{i}: \pi_{n+1}\left(X_{i}, x_{i}\right) \rightarrow H_{n+1}\left(X_{i}\right), j \in J$, and $\eta_{n}$ is the limit Hurewicz homomorphism in the sense of Kuperberg [9, p. 26]. The exactness of the top row of diagram (3.1) follows from Theorem 2 of Watanabe [15] (cf. Grossman [5] or Edwards and Hastings [4, 5.2.1]). Milnor [11, Theorem 4] proved the bottom row of (3.1) is exact. Since $(X, x)$ is approximatively $k$-connected for $k=0,1, \ldots, n-1$, by [9, Theorem 3.2] $\eta_{n}$ is an isomorphism. It remains to prove that $\mu$ is an isomorphism. To show it, note that we may assume every $\left(X_{i}, x_{i}\right), i \in J$, is $(n-1)$-connected. (This is proved by the same way as in Lemma (1.6) of Nowak [12].) Then the Hurewicz homomorphism $\mu_{i}: \pi_{n+1}\left(X_{i}, x_{i}\right) \rightarrow H_{n+1}\left(X_{i}\right)$ is onto by [6, Theorem 2.6]. Let $G_{i}=\operatorname{Kernel} \mu_{i}, i \in J$. Then $\left\{G_{i}\right\}$ forms an inverse sequence. Consider the following exact sequence in the category pro- $G$ of pro-groups:

$$
0 \rightarrow\left\{G_{i}\right\} \stackrel{\left\{j_{i}\right\}}{\rightarrow}\left\{\pi_{n+1}\left(X_{i}, x_{i}\right)\right\} \rightarrow\left\{H_{n+1}\left(X_{i}\right)\right\} \rightarrow 0,
$$

where $j_{i}: G_{i} \rightarrow \pi_{n+1}\left(X_{i}, x_{i}\right)$ is the inclusion homomorphism, $i \in J$, and 0 means a zero object in pro- $G$. By $[3$, p. 256] the sequence (3.2) induces the exact sequence:

$$
\lim _{\leftarrow}^{(1)}\left\{G_{i}\right\} \stackrel{\lim \left\{j_{i}\right\}}{\rightarrow} \lim ^{(1)}\left\{\pi_{n+1}\left(X_{i}, x_{i}\right)\right\} \rightarrow \lim _{\leftarrow}^{(1)}\left\{H_{n+1}\left(X_{i}\right)\right\} \rightarrow 0 .
$$

Let $f:\left(S^{n+1}, s\right) \rightarrow\left(X_{i}, x_{i}\right)$ be a map representing an element $\alpha \in \pi_{n+1}\left(X_{i}, x_{i}\right)$. Then $\alpha \in G_{i}$ if and only if $f$ is $n$-deformable. From this fact and the $(n+1, n)$-movability of $(X, x)$ it follows that the inverse sequence $\left\{G_{i}\right\}$ satisfies the Mittag-Leffler condition. Thus $\lim _{\leftarrow}^{(1)}\left\{G_{i}\right\}=0$ by [3, p. 256]. The 
exactness of the sequence (3.3) shows that $\mu$ is an isomorphism. This completes the proof.

COROllary 2. Let $(X, x)$ be a pointed movable compactum such that $\underline{\pi}_{i}(X, x)=0$ for $i=0,1, \ldots, n-1, n>1$. Then $\underline{\pi}_{n}(X, x)=\underline{\underline{\pi}}_{n}(X, x)=$ $\check{H}_{n}(X)={ }^{s} H_{n+1}(X)$.

This is obvious from Corollary 1, Theorem 2 and Borsuk [2, Chapter V, Theorem (10.1)].

Corollary 3. Let $(X, x)$ be approximatively connected for $k=0,1, \ldots, n$ - 1. If there exists an inverse sequence $\left\{\left(X_{i}, x_{i}\right)\right\}$ such that $(X, x)=\lim \left(X_{i}, x_{i}\right)$ and $\lim ^{(1)}\left\{\pi_{n}\left(X_{i}, x_{i}\right)\right\}=0$, then $\xi_{n}: \underline{\pi}_{n}(X, x) \rightarrow^{s} H_{n+1}(X)$ is an isomorphism.

Proof. By [8], $(X, x)$ is pointed $S^{n}$-movable. Since the approximative $k$-connectedness for $k=0,1, \ldots, n-1$ and the pointed $S^{n}$-movability imply the pointed $n$-movability, the corollary follows from Lemma 1 and Theorem 2.

COROLlaRY 4. Let $X$ be an $S^{n}$-like continuum and let $n \neq 2$. Then, for every point $x \in X$, the homomorphism $\xi_{n}: \underline{\underline{\pi}}_{n}(X, x) \rightarrow{ }^{s} H_{n+1}(X)$ is an isomorphism.

Proof. By (2.3) of Example $1,(X, x)$ is $(n+1, n)$-movable. Thus, if $n>2$ the corollary follows from Theorem 2. For $n=1$, consider the diagram (3.1). Obviously $\lim _{\leftarrow}^{(1)}\left\{\pi_{2}\left(X_{i}, x_{i}\right)\right\}=\lim _{\leftarrow}^{(1)}\left\{H_{2}\left(X_{i}\right)\right\}=0$ and $\eta_{1}$ is an isomorphism. Thus $\xi_{1}$ is an isomorphism.

Corollary 5. Let $X$ be an $S^{2}$-like continuum and let $x \in X$. Then the following conditions are equivalent:

(3.4) $(X, x)$ is pointed movable.

(3.5) $\xi_{2}: \underline{\pi}_{2}(X, x) \rightarrow{ }^{s} H_{3}(X)$ is an isomorphism.

Proof. The implication (3.4) $\rightarrow(3.5)$ is a consequence of Theorem 2. To prove (3.5) $\rightarrow(3.4)$, assume that $(X, x)$ is not pointed movable. Let $\left\{\left(X_{i}, x_{i}\right)\right.$, $\left.p_{i, i+1}\right\}$ be an inverse sequence consisting of 2-spheres such that $(X, x)$ $=\lim \left(X_{i}, x_{i}\right)$. By Mardešić and Segal [10, Theorem 4], $X$ is neither of trivial shape nor of the shape of $S^{2}$. Then, for an infinite number of $i \in J$, the bonding map $p_{i, i+1}$ has degree $\geqslant 2$. Since $\pi_{3}\left(X_{i}, x_{i}\right)=Z$, we have $\lim ^{(1)}\left\{\pi_{3}\left(X_{i}, x_{i}\right)\right\} \neq 0$. On the other hand, $\lim ^{(1)}\left\{H_{3}\left(X_{i}\right)\right\}=0$. Thus the homomorphism $\mu$ in (3.1) is not an isomorphism. Hence $\xi_{2}$ is not an isomorphism.

Example 1 and Corollary 5 imply that we cannot omit the $(n+1, n)$ movability or the approximative connectivity of $(X, x)$ in Theorem 2 . Finally, we give an example of a pointed continuum $(X, x)$ such that $(X, x)$ is approximatively 1 -connected but not pointed $(3,2)$-movable, and $\xi_{2}$ is an isomorphism.

EXAMPLE 2. For $n \in J$, let $X_{n}$ be a one point union of a 2-sphere $S_{n}$ and 
3-spheres $S_{n, i}, i=1,2, \ldots, n$, with the base point $x_{n}$. Let $p_{n, n+1}:\left(X_{n+1}, x_{n+1}\right)$ $\rightarrow\left(X_{n}, x_{n}\right)$ be a map such that

$p_{n, n+1} \mid S_{n+1}$ is a map from $S_{n+1}$ to $S_{n}$ with degree 2,

$p_{n, n+1} \mid S_{n+1,1}$ is the Hopf map from $S_{n+1,1}$ to $S_{n}$,

$p_{n, n+1} \mid S_{n+1, i}, i=2, \ldots, n+1$, is a homeomorphism from $S_{n+1, i}$ to $S_{n, i-1}$.

Let $(X, x)$ be the limit of the inverse sequence $\left\{\left(X_{n}, x_{n}\right), p_{n, n+1}\right\}$. As shown in Example $1,(X, x)$ is not $(3,2)$-movable. Obviously $\underline{\pi}_{2}(X, x)=\check{H}_{2}(X)=0$. Since both of the inverse sequences $\left\{\pi_{3}\left(X_{n}, x_{n}\right)\right\}$ and $\left\{H_{3}\left(X_{n}\right)\right\}$ satisfy the Mittag-Leffler condition, by diagram (3.1) we have $\underline{\underline{\pi}}_{2}(X, x)={ }^{s} H_{3}(X)=0$.

\section{REFERENCES}

[1] K. Borsuk, On the n-movability, Bull. Acad. Polon. Sci. Sér. Sci. Math. Astronom. Phys. 20 (1972), 859-864.

[2] __ Theory of shape, PWN-Polish Scientific Publishers, Warsaw, 1975.

[3] A. K. Bousfield and D. M. Kan, Homotopy limits, completions and localizations, Lecture Notes in Math., vol. 304, Springer-Verlag, Berlin and New York, 1972.

[4] D. A. Edwards and H. M. Hastings, Cech and Steenrod homotopy theories with applications to geometric topology, Lecture Notes in Math., vol. 542, Springer-Verlag, Berlin and New York, 1976.

[5] J. Grossman, Homotopy classes of maps between pro-spaces, Michigan Math. J. 21 (1974), 355-362.

[6] P. J. Hilton, An introduction to homotopy theory, Cambridge Univ. Press, London and New York, 1953.

[7] Y. Kodama and T. Watanabe, A note on Borsuk's n-movability, Bull. Acad. Polon. Sci. Sér. Sci. Math. Astronom. Phys. 22 (1974), 289-294.

[8] A. Koyama, J. Ono and K. Tsuda, An algebraic characterization of pointed $S^{n}$-movability, Bull. Acad. Polon. Sci. Sér. Sci. Math. Astronom. Phys. 25 (1977), 1249-1252.

[9] K. Kuperberg, An isomorphism theorem of the Hurewicz type in Borsuk's theory of shape, Fund. Math. 77 (1972), 21-32.

[10] S. Mardešić and J. Segal, Movable compacta and ANR-systems, Bull. Acad. Polon. Sci. Sér. Sci. Math. Astronom. Phys. 18 (1970), 649-654.

[11] J. Milnor, On the Steenrod homology, Mimeographed notes, Berkeley, 1960.

[12] S. Nowak, On the fundamental dimension of approximatively 1-connected compacta, Fund. Math. 89 (1975), 61-79.

[13] J. B. Quigley, An exact sequence from the nth to the $(n-1)$-st fundamental group, Fund. Math. 77 (1973), 195-210.

[14] N. E. Steenrod, Regular cycles of compact metric spaces, Ann. of Math. 41 (1940), 833-851.

[15] T. Watanabe, On a problem of Y. Kodama, Bull. Acad. Polon. Sci. Sér. Sci. Math. Astronom. Phys. 25 (1977), 981-986.

Department of Mathematics, University of Tsukuba, Ibaraki, JaPan 